\title{
Die Werteorientierung beginnt mit einem beruflichen "Grundlagenvertrag“
}

In diesem „Grundlagenvertrag“ können Sie ihn als Führungskraft z. B. mithilfe der sechs skizzierten Regeln neu gestalten. Sie können die aus Ihrer Sicht wichtigsten drei bis maximal sechs Regeln mit Ihren Mitarbeitern als gemeinsame Grundlage Ihrer Zusammenarbeit anbieten und mit ihnen die Funktionalität in einer eigenen Testphase überprüfen und gegebenenfalls gemeinsam verändern. Es ist für die laufende Zusammenarbeit entscheidend, dass Sie eine gemeinsam akzeptierte und tragfähige Vereinbarung mit Ihren Mitarbeitern treffen, für die alle Mitarbeiter die beschriebene Verantwortung übernehmen.

In dieser Vereinbarung werden die Werte Respekt, Vertrauen, Sicherheit, Gerechtigkeit, Toleranz und Verantwortlichkeit hervorgehoben.

1. Ich gehe mit anderen respektvoll um

2. Ich spreche für mich

3. Ich frage nach, statt nur auf meine Vermutungen zu reagieren - die sich selbst erfüllenden Prophezeiungen

4. Ich höre zu und lasse den anderen ausreden

5. Ich trage selbst die Verantwortung für mein Handeln und für meinen Beitrag zum Erfolg unserer Zusammenarbeit

6. Ich verhalte mich vertrauenswürdig und lasse alle persönlich zurechenbaren Informationen in unserem Team

Alle Regeln, die Sie mit Menschen privat oder beruflich vereinbaren und nicht beachten, schaden sowohl Ihrer Vorbildfunktion als auch dem Ihnen entgegengebrachten Vertrauen und Respekt. Sie wirken sich ebenfalls zwangsläufig auf die Zusammenarbeit mit Ihren Mitarbeitern oder auf das Zusammenleben in Ihrer Familie oder Ihrem Freundeskreis aus. 